\title{
Reavaliando o Bem Sex-Role Inventory
}

\section{Reevaluating the Bem Sex-Role Inventory}

\author{
José Augusto Evangelho HERNANDEZ
}

\begin{abstract}
Resumo
O objetivo deste estudo foi avaliar a estrutura do Bem Sex Role Inventory respondido por uma amostra não probabilística de homens e mulheres brasileiras. Mais de uma década após a última revisão brasileira do inventário, um novo exame faz-se necessário, devido à forte influência cultural sobre seus itens. A escala foi aplicada junto a 922 sujeitos, recrutados em diversos locais de Porto Alegre (RS) e região metropolitana. Foi realizada uma avaliação da estrutura da escala usando a técnica estatística análise fatorial. Além disso, foi examinada a fidedignidade do instrumento. Os resultados obtidos foram comparados com os estudos anteriores. Em geral, apareceram evidências que dão forte apoio à versão verificada e, desta forma, habilitam-na para ser usada na pesquisa psicológica.
\end{abstract}

Unitermos: Avaliação psicológica. Gênero. Papéis sexuais.

\begin{abstract}
The aim of this study was to evaluate the structure of the Bem Sex-Role Inventory, answered by a non-probabilistic sample of Brazilian men and women. More than a decade after the last Brazilian review of the Inventory, it has become necessary to conduct a fresh examination, due to the strong cultural influence regarding its content. The scale was applied to 922 subjects recruited from several locations in Porto Alegre (in the state of Rio Grande do Sul) and its metropolitan area. An evaluation of the structure of the scale was carried out using Factorial Analysis. In addition, the reliability of the scale was also examined. The findings were compared to previous studies. Generally speaking, the evidence strongly supports the approved version, thereby qualifying it to be used in psychological research.
\end{abstract}

Uniterms: Psychological assessment. Gender. Sex role.

O objetivo deste trabalho foi examinar a adaptação de Hutz e Koller (1992) para o Bem Sex Role Inventory (BSRI) (Bem, 1974), instrumento construído com a finalidade de avaliar papéis sexuais. Recorrendo à técnica estatística análise fatorial, o estudo explorou a estrutura latente das escalas feminina, masculina e neutra do inventário, observando o desempenho dos itens na função de representação dos construtos. Como esses elementos estão submetidos a uma forte influência cultural, é recomendável que, periodicamente, sejam revisados para verificar se continuam refletindo as mudanças sociais. Além disso, foram calculados os coeficientes de fidedignidade para cada uma das escalas do Bem Sex Role Inventory (BSRI).

A revisão de Constantinople (1973), marco histórico nesta área de investigação, concluiu que masculinidade e feminilidade parecem estar entre os inúmeros conceitos turvos do vocabulário dos psi-

\footnotetext{
४े
}

1 Instituição Educacional São Judas Tadeu, Curso de Educação Física. R. Dom Diogo de Souza, 100, Cristo Redentor, $91350-000$, Porto Alegre, RS, Brasil. E-mail: <hernandz@portoweb.com.br>. 
cólogos. A mais generalizada e abrangente definição desses termos é a de que são características relativamente duradouras, mais ou menos enraizadas na anatomia, fisiologia e experiências iniciais e que, efetivamente, servem para distinguir homens e mulheres na aparência, atitudes e comportamento.

Lenney (1991) comentou que, a despeito da enorme popularidade desses construtos básicos (papéis masculinos e femininos), os investigadores parecem evitar uma definição clara dos mesmos, tanto conceitual quanto operacionalmente. Alguns propuseram o uso de expressões que se relacionam a vários aspectos estritos dos papéis sexuais, resultando em uma grande proliferação de palavras, o que gerou mais confusão. Por exemplo, alguns definiram orientação de papel sexual como uma percepção subjacente, não necessariamente consciente, de masculinidade e feminilidade do self. Outros afirmaram que medidas de orientação de papel sexual tentam distinguir entre papéis tradicionais e não tradicionais relacionados ao sexo, atividades, carreiras e estilos de vida.

Além disso, Gilbert (1985) sustentou que papéis sexuais referem-se às expectativas normativas sobre a divisão de trabalho entre os sexos e as regras sobre interações sociais relativas a gênero que existem em uma cultura ou contexto histórico particular.

Nos anos pré-científicos da psicologia, acreditava-se fortemente na base constitucional e nos instintos como determinantes das diferenças sexuais nas atitudes e nos comportamentos humanos. Na atualidade, sabe-se que muito antes de perceberem as diferenças sexuais anatômicas, os meninos e as meninas recebem a informação de que há dois tipos de pessoas no mundo social: homens e mulheres (Gerrig \& Zimbardo, 2005). Por que as mulheres tendem a comportar-se de certas maneiras e os homens de outras? Oliveira (1983) já observava que o sistema social parece levar meninos e meninas a tomarem rumos distintos na direção de como ser homem e mulher, respectivamente.

Até os anos 60, os pesquisadores procuraram avaliar aspectos masculinos e femininos baseados em um modelo bipolar e unidimensional. Nessa perspectiva, o paradigma central é o pressuposto de que homens e mulheres diferem nos estereótipos sociais, nos padrões de desempenho de papéis sexuais e nas prescrições o feminino. Por exemplo, homens devem ser mais independentes e agressivos, enquanto as mulheres, mais dependentes e amáveis. Entretanto, Oliveira (1983) também referiu que, nos anos 60 e 70, muito foi escrito sobre as mudanças desses papéis sexuais, com homens apresentando emoções e mulheres sendo mais assertivas e negociadoras.

Várias teorias psicológicas dedicaram-se à tentativa de explicar o desenvolvimento de papéis sexuais. Entre as mais importantes estão a psicanálise, a aprendizagem social e a psicologia cognitiva.

Na abordagem psicanalítica, a evolução e a resolução do conflito edípico são as responsáveis pela identificação das crianças com as características, atitudes e comportamentos dos pais de mesmo sexo biológico. Segundo Fast (1993), Freud postulava que a diversidade de personalidade entre homens e mulheres é o resultado de uma sequência invariável de estágios (oral, anal e fálico) no desenvolvimento psicológico da criança.

Na teoria da aprendizagem social surgiram duas grandes correntes, uma que prescinde da referência aos processos intrapsíquicos e enfatiza a interação entre a conduta e os eventos ambientais na aquisição de comportamentos, e outra que concede grande importância aos processos cognitivos que medeiam a aquisição das condutas sexualmente tipificadas (Lott \& Maluso, 1993).

Na primeira corrente, os comportamentos sexualmente tipificados são vistos como os que proporcionam uma distinta gratificação a um e outro sexo, ou têm consequências que variam segundo o sexo do sujeito. A tipificação sexual é o processo no qual o indivíduo adquire padrões de comportamento vinculados ao sexo. Primeiro, ele aprende a distinguir esses padrões, depois, a generalizar essas experiências concretas a situações novas e, finalmente, a usar o comportamento. A ação do condicionamento operante pode ser observada quando a criança é recompensada ou punida por assumir um comportamento adequado ou inadequado ao próprio sexo. Meninos são frequentemente reprimidos por chorar, brincar com bonecas ou mostrar interesse por atividades domésticas (cozinhar, lavar louça, lavar e passar roupas). Da mesma forma, meninas são criticadas por serem agressivas ou estabanadas. De formas sutis, todas as pessoas estão permanentemente encarregadas de recompensar ou punir essas crianças 
pelo desempenho em comportamentos julgados mais ou menos apropriados aos seus sexos (Lott \& Maluso, 1993).

Na segunda corrente, estudos empíricos mostraram que aprendizagem vicária é uma das fontes mais importantes de aquisição de comportamentos sexuais (Ross, Anderson \& Wisocki, 1982). Algumas pesquisas têm encontrado que não necessariamente meninos imitam modelos masculinos e meninas imitam modelos femininos, mas isso pode ser resultado de uma falha na identificação do comportamento. Alguns investigadores têm apontado que primeiro as crianças precisam aprender que o comportamento observado é desempenhado mais frequentemente por um sexo em específico (Bussey \& Bandura, 1984).

As teorias cognitivas tradicionais - genética evolutiva (Kohlberg, 1972) e processamento de informações (Bem, 1981) - convivem em um grupo denominado psicologia cognitiva e também abordaram o desenvolvimento dos papéis sexuais.

Kohlberg (1972) sustentou que o processo de tipificação sexual apoia-se no marco evolutivo geral da compreensão da realidade, afetando a organização cognitiva dos sexos sobre a qual se conformará, gradativamente, a constância do gênero. Influenciado pela visão piagetiana, o autor defendeu que as mudanças na maturação cognitiva afetam a autopercepção e refletem-se na categorização de estereótipos e valores sobre o sexo.

Na teoria de processamento de informação foram elaborados alguns modelos que explicam o desenvolvimento e funcionamento dos estereótipos de gênero com base em esquemas cognitivos integráveis no autoconceito. Se os sujeitos integram a informação recebida sobre uma base de esquemas previamente estabelecidos, é lógico pensar que a designação social de gênero atuará prontamente, possibilitando o desenvolvimento de uma extensa rede de associações internas que, ativadas, mais adiante serão decisivas para interpretar a realidade e, especialmente, o conceito sobre si mesmo (Barberá, 1998).

Para Sternberg (2000), conceito é a unidade essencial do conhecimento simbólico, a ideia que se tem sobre algum objeto. Um conceito também está relacionado a muitos outros, por exemplo, mulher com delicadeza, suavidade, afetividade. Os conceitos, em geral, estão organizados em esquemas. Os esquemas são estruturas mentais que representam o conhecimento e são constituídos por uma série de conceitos inter-relacionados de forma significativa.

Em suas primeiras teorizações, Bem (1974) considerou masculinidade e feminilidade como grupos complementares de características e comportamentos positivos. A Gender Schema Theory (Bem, 1981) ocupou um lugar de destaque no contexto das abordagens cognitivas de processamento de informação. O esquema de gênero foi concebido como um contínuo singular, com dois pólos; em um deles estariam situados os sujeitos tipificados sexualmente (altamente masculinos ou altamente femininos), enquanto no extremo oposto estariam os sujeitos com orientações fracas de papéis sexuais e os que apresentam tendências de sexo cruzado, não esquemáticos segundo o gênero ou não tipificados sexualmente. A classificação desses dois tipos (esquemáticos e não esquemáticos) baseou-se nas divergências quanto à disponibilidade cognitiva, que se manifestaria tanto em nível de discriminação perceptiva, associativa e de memória, quanto no que se refere às expectativas e crenças sobre a polaridade de gêneros. Os esquemáticos teriam maior predisposição para classificar as informações nas categorias masculino e feminino e para decidir quais atributos incluiriam ou não em seu autoconceito.

Bem (1981) propôs a ideia de um esquema cognitivo de gênero que estaria estreitamente ligado aos padrões socioculturais de comportamentos esperados para cada um dos sexos. Uma vez aprendido este esquema, isso predisporia a criança a perceber o mundo também em termos sexuais.

Os esquemas de gênero servem para a criança avaliar a si própria e aos que a rodeiam em termos de adequação aos padrões definidos pela sociedade para os sexos, sentindo-se motivada a ajustar-se a estas definições. Assim, autoestima e autoconceito são desenvolvidos sob a regência do esquema de gênero. Quando o indivíduo percebe a sua própria conformidade a um padrão tradicional, a diferenciação do autoconceito baseada em distinções de sexo é fortalecida e resulta em uma identidade de papel sexual tradicional.

Em oposição, tais conotações não são marcantes para pessoas não tipificadas ou não esquemáticas. $\mathrm{O}$ conteúdo do que constitui os domínios da mascu- 
linidade e da feminilidade não é enfatizado, mas sim o tipo de processo cognitivo. Segundo este ponto de vista, a androginia representa uma forma particular de processar informação. Os andróginos não são esquemáticos e, portanto, não contam com conotações sexuais para orientar o seu processamento de informação. Todavia, aqui não haveria a ideia de hierarquia contida na abordagem evolutiva, na qual a androginia seria definida como uma condição superior a ser atingida. Para Bem (1981), ela seria simplesmente uma forma diferente de processamento das informações disponíveis no meio.

Jönsson e Carlsson (2000) encontraram relação significativa entre os sujeitos classificados com andróginos no BSRI e a criatividade. Além disso, a noção de flexibilidade de papel sexual como uma característica dos andróginos tem sido proposta há mais de três décadas (Bem, 1974). Flexibilidade de papel sexual tem sido conceitualizada como uma qualidade adaptativa relativa à apresentação de comportamentos flexíveis às situações. O estudo de Cheng (2005) chegou à conclusão de que os andróginos podem não ter um conhecimento mais amplo de estratégias de coping do que outros, porém são mais flexíveis na distribuição destas conforme a necessidade de controle de diferentes estressores. A autora também verificou que andróginos, em períodos estressantes do ciclo vital, experimentam níveis mais baixos de depressão.

Teorias defendem que a androginia denota habilidade para integrar as facetas da personalidade (Bem, 1977). Desta forma, na tomada de decisão sobre como responder à realidade, o aspecto adaptativo da flexibilidade de papel sexual poderia ser representado pela integração do pensamento pessoal orientado à situação. Os tipificados masculinos e femininos tendem a dar mais atenção às estratégias de coping apropriadas aos seus sexos e ignoram amplamente as características das mudanças do ambiente.

Diversas escalas foram desenvolvidas para medir orientação de papéis sexuais, e as duas mais usadas são o BSRI de Bem (1974) e o Personal Attributes Questionnaire (PAQ) de Spence, Helmreich e Stapp (1975). Na forma original, o BSRI caracterizava uma pessoa como masculina, feminina ou andrógina de acordo com algumas características de personalidade vinculadas aos papéis que as dimensões da masculinidade e da feminilidade são empírica e logicamente independentes; o conceito de androginia psicológica é fidedigno e está definido operacionalmente pela obtenção de índices altos ou baixos em ambas as escalas de masculinidade e feminilidade. Escores muito tipificados sexualmente não refletem uma tendência geral do indivíduo para responder em uma direção socialmente desejável, mas para a autodescrição em concordância com padrões de comportamento desejáveis para homens e mulheres.

O ajustamento psicológico e o relacionamento interpessoal são as duas maiores áreas associadas à pesquisa em papéis sexuais. O trabalho inicial de Bem (1974) anunciou a ideia de que a maioria das pessoas bem ajustadas é andrógina, possuindo qualidades femininas e masculinas. Isto contrasta com o modelo anterior de congruência, ou seja, homens masculinos e mulheres femininas compondo a maioria da população bem ajustada.

O BSRI foi revisado por Bem (1977) após as contribuições de Spence et al. (1975). Estes argumentaram que as pessoas classificadas como andróginas deveriam apresentar índices altos nas escalas de masculinidade e feminilidade. Pessoas com índices baixos em ambas as escalas deveriam ser classificadas como indiferenciadas.

Ainda nos anos 70, o instrumento de Bem (1974) foi submetido a vários testes. Whetton e Swindells (1977) apresentaram uma análise fatorial dos itens do BSRI que gerou alguma evidência da independência das dimensões masculinidade e feminilidade. Esses dois fatores juntos foram responsáveis por 17\% da variância; no entanto, diversos outros fatores também foram extraídos. Os autores concluíram que duas dimensões apenas poderiam significar uma visão simplista para um inventário de papéis sexuais.

Nas duas décadas subsequentes, o BSRI sofreu uma verdadeira avalanche de avaliações psicométricas em contextos culturais diferentes. Em alguns deles os resultados não corroboraram as suposições de Bem (1977). Por exemplo, Heerboth e Ramanaiah (1985) testaram a proposta de que as escalas masculinas e femininas medem padrões de comportamentos tipificados sexualmente, desejáveis para homens e mulheres na cultura americana. Os resultados não apoiaram as suposições de Bem. Da mesma forma, para Ward e Sethi (1986) o BSRI não foi uma medida válida de androginia psico- 
lógica na Malásia e sul da Índia, provavelmente devido às diferenças culturais nos construtos de masculinidade e feminilidade. Maznah e Choo (1986) aplicaram o BSRI a uma amostra não ocidental e os resultados da análise fatorial confirmaram a ideia de que o inventário é multidimensional.

Hiller e Philliber (1985), alegando que a maioria das avaliações do BSRI baseava-se em amostras universitárias, administraram o instrumento a sujeitos casados. As escalas masculina e feminina apresentaram correlações com ocupações sexualmente tipificadas, participação no trabalho doméstico, atitudes sobre quem deve fazer o trabalho doméstico ou ser o provedor financeiro, educação e idade. Os resultados reforçaram o argumento de que o conteúdo dos estereótipos sexuais pode estar intimamente associado aos papéis sociais. Na mesma linha, Schmitt e Millard (1988) encontraram evidências empíricas que apoiaram a teoria de esquema de gênero, isto é, o BSRI foi uma medida válida para discriminar indivíduos esquemáticos e não esquemáticos.

O estudo de Wilson et al. (1990) investigou a validade do BSRI em professores estagiários no Zimbabwe. Os dados revelaram elevados índices de confiabilidade para as escalas masculina e feminina. A análise fatorial mostrou uma estrutura bem definida de fatores masculinos e femininos, confirmando a validade de construto do instrumento naquela cultura. Da mesma forma, o objetivo de Katsurada e Sugihara (1999) foi validar o BSRI na cultura japonesa. Os resultados, no geral, mostraram que o instrumento foi válido para o Japão. Contudo, os autores recomendaram que alguns adjetivos do inventário fossem substituídos para melhorar esta validade com japoneses.

Twenge (1997) realizou a primeira meta-análise sobre as mudanças de percepção das características masculinas e femininas, abrangendo os vinte anos subsequentes à criação do BSRI. O trabalho compreendeu 63 amostras examinadas por este instrumento. Foi constatado que, na escala masculina, o tamanho do efeito para as diferenças sexuais teve um decréscimo significativo ao longo do tempo. Mudanças encontradas no inventário indicaram um crescimento dos escores das mulheres nos traços masculinos estereotipados. No entanto, o mesmo não aconteceu com homens em relação aos estereótipos femininos. Esses resultados sugeriram que mudanças culturais podem afetar a personalidade das pessoas.

Reed-Sanders, Dodder e Webster (2001) utilizaram o BSRI para examinar sujeitos mexicano-americanos, mexicanos e ingleses, estudantes de universidades americanas ou mexicanas. Os pesquisadores concluíram que, independentemente da cultura, o inventário revelou que os escores dos homens foram mais elevados em masculinidade do que os das mulheres em feminilidade, o que confirmou a validade do BSRI para discriminar os sexos. Entretanto, na amostra mexicana houve uma proporção maior de respostas na categoria indiferenciada, o que foi interpretado pelos autores como uma provável limitação do inventário para identificar características masculinas e femininas nessa cultura.

Zhang, Norvilitis e Jin (2001) também fizeram uma comparação do desempenho do BSRI entre culturas. O inventário foi aplicado a uma amostra universitária de americanos e chineses. As análises fatoriais mostraram diferentes padrões entre as culturas. Os resultados indicaram que apenas 40\% dos itens das escalas masculina e feminina foram equivalentes entre as culturas examinadas. Além disso, os escores dos chineses foram, em geral, menores que os dos americanos.

Konrad e Harris (2002) investigaram se os itens do BSRI refletiam uma visão atualizada sobre gênero. Esses pesquisadores encontraram que homens urbanos, tanto americanos quanto afro-americanos, fizeram avaliações mais tradicionais de papéis sexuais, enquanto mulheres urbanas euro-americanas expressaram visões mais liberais. Na avaliação das mulheres euro-americanas, apenas quatro dos 40 itens das escalas masculina e feminina foram significativamente diferentes para os sexos.

A investigação de Choi e Fuqua (2003) revisou 23 estudos de validação do BSRI localizados nas bases de dados ERIC e PsycLit. Todos os trabalhos examinados usaram análise fatorial exploratória e foram conduzidos nos 25 anos seguintes à publicação do instrumento. Na maioria dos casos revistos, as análises extraíram de dois a quatro fatores, e os masculinos apresentaram maior complexidade. Para os autores, esses dados sugeriram que masculinidade e feminilidade não estavam operacionalizadas de forma adequada no inventário. $\mathrm{Na}$ conclusão, os pesquisadores levantaram a possibilidade 
de o BSRI não capturar a natureza complexa da masculinidade e da feminilidade.

Özkan e Lajunen (2005) avaliaram as escalas do BSRI entre universitários turcos, usando uma versão reduzida do instrumento. Na análise fatorial foi identificada, para homens e mulheres, uma estrutura similar à original de Bem (1981). A consistência interna das escalas masculina e feminina foi aceitável. Além disso, na escala masculina foram apuradas diferenças entre as respostas de homens e mulheres em apenas 20\% dos itens, enquanto na escala feminina foram constatadas diferenças em $80 \%$ dos itens.

Apesar dos trinta anos de existência, o BSRI continua sendo o principal instrumento para medir papéis sexuais, reconheceu Peng (2006). Esse pesquisador realizou um estudo das propriedades psicométricas da versão reduzida do inventário com uma amostra de Taiwan. Os dados revelaram consistência interna satisfatória e, ao mesmo tempo, uma estrutura de masculinidade e feminilidade mais complexa do que o prescrito para o instrumento. Consequentemente, o autor sugeriu cautela no uso do instrumento antes que sejam efetuadas novas adaptações do mesmo à cultura local.

O primeiro processo de adaptação do BSRI à cultura brasileira foi realizado por Oliveira (1982). No entanto, uma avaliação desta feita por Koller, Hutz, Vargas e Conti (1990) revelou diversos problemas, que levaram a uma readaptação do instrumento (Hutz \& Koller, 1992), em um trabalho que compreendeu dois estudos.

No primeiro estudo, realizado com 87 universitários, Hutz e Koller (1992) fizeram uma avaliação do grau de estereotipia sexual de 150 adjetivos, com a finalidade de compor os sessenta itens para as escalas feminina, masculina e neutra do BSRI readaptado. No segundo estudo, com 416 universitários, os pesquisadores examinaram a fidedignidade e a consistência da readaptação brasileira do instrumento. Esses investigadores encontraram alguns problemas na estrutura fatorial do inventário: diversos itens que, pelo que se esperava, carregariam em um determinado fator, acabaram carregando em outros. Além disso, ocorreu uma grande dispersão dos itens da escala feminina, o que revelou a fragilidade desta. Por outro lado, como os autores constataram que a maioria dos itens da escala masculina foi extraída como fator principal, concluíram 78 que ela apresentou as melhores condições psico- métricas. Ao final, os pesquisadores consideraram a versão avaliada fidedigna. Os Alfas de Cronbach calculados foram os seguintes: escala masculina 0,81, escala feminina 0,80 e escala neutra 0,58.

Formiga e Camino (2001) estudaram as dimensões de masculinidade e feminilidade no BSRI. Esses autores declararam utilizar a versão de Hutz e Koller (1992), que manteve os mesmos sessenta itens da original (Bem, 1974), embora tenham apresentado o instrumento composto por 18 adjetivos. Pode-se deduzir que Formiga e Camino usaram uma forma reduzida do inventário, visto que o relato não é claro neste aspecto. Em suma, mediante análise fatorial e escala multidimensional, os autores reconheceram a competência do BSRI para medir estereótipos vinculados ao sexo no Brasil.

O objetivo do estudo aqui descrito foi abordar novamente a versão de Hutz e Koller (1992) com uma amostra de perfil diferente, uma década depois, para reexaminar a estrutura do BSRI. Conforme visto na literatura, são recomendadas revisões periódicas sistemáticas do inventário, considerando a forte influência da cultura sobre os itens que representam seus construtos, os quais podem variar conforme as mudanças da sociedade.

\section{Método}

\section{Participantes}

Foi feita uma amostra não probabilística, de conveniência. Os participantes foram abordados em diversos locais de Porto Alegre (RS) e região metropolitana. Participaram deste estudo 922 indivíduos: 503 mulheres e 419 homens. A idade dos participantes variou de 14 a 74 anos, média de 31,2 anos. Quanto à escolaridade, 41 sujeitos tinham ensino fundamental, 244 tinham ensino médio, 560 tinham ensino superior, 68 tinham pós-graduação e 8 não forneceram esta informação.

\section{Instrumentos}

Foi utilizado o Bem Sex Role Inventory (BSRI), de Bem (1974), adaptado por Oliveira (1982) e readaptado por Hutz e Koller (1992).

Este instrumento de avaliação de papéis sexuais é um conjunto de sessenta itens de adjetivos que 
compõem três escalas: masculina, feminina e neutra. Os vinte itens da escala masculina são: 1, 4, 7, 10, 13, 16, $19,22,25,28,31,34,37,40,43,46,49,52,55,58$. Os vinte itens da escala feminina são: 2, 5, 8, 11, 14, 17, 20, 23, 26, $29,32,35,38,41,44,47,50,53,56,59$. Os vinte itens da escala neutra são os restantes (Tabela 1). Os respondentes usaram uma escala tipo Likert de sete pontos ( $1=$ a característica nunca é verdadeira; 7 = a característica sempre é verdadeira) para avaliar os adjetivos em relação às suas próprias características pessoais percebidas.

\section{Procedimentos}

O instrumento foi respondido em locais diversos, tais como praças, shoppings, salas de aula, pátios de escolas, campi de universidades, reuniões de pais (entrega dos boletins dos filhos), reuniões de casais e em residências particulares. OTermo de Consentimento Livre e Esclarecido foi lido e assinado por todos os participantes após receberem informações sobre os objetivos da pesquisa. Algumas vezes, os dados foram coletados em grupo e, em outros casos, individualmente.

Os dados foram analisados pelo Statistical Package of Social Sciences (SPSS), versão 11.5, com estatísticas descritivas, análise de variância e análise fatorial. A análise fatorial da escala forneceu informações sobre a estrutura interna, sobre o peso dos fatores e a complexidade estrutural dos itens. Como os itens femininos e masculinos foram criados para serem ortogonais, a solução fatorial foi rotada usando-se o método varimax. Para a rotação foi determinado o número de três fatores, previsto por Bem (1974); contudo, antes foi executada uma solução inicial com extração de fatores com autovalores maiores que 1,0. Além disso, a fidedignidade do BSRI e de suas escalas foi avaliada pelo Coeficiente Alfa de Cronbach.

\section{Resultados}

O teste Kaiser-Meyer-Olkin revelou uma medida de adequacidade amostral de 0,89, considerada meritosa pela classificação de Kaiser e Rice (1974), ou seja, acima de 0,80 . A solução inicial da análise fatorial para componentes principais extraiu 13 fatores com autovalores maiores que 1, responsáveis por 55,77\% da variância (Kaiser, 1960).
O Scree Test de Cattell (1966), critério para determinar o número de fatores, revelou que soluções de três a seis fatores eram possíveis. Por outro lado, pareceu razoável estabelecer o ponto de corte entre o terceiro e o quarto fatores extraídos, visto que apareceu um decréscimo de 4 para 2 autovalores entre ambos (Figura 1).

Sem dúvida, a solução para três fatores (responsáveis por 31,74\% da variância total) com rotação varimax mostrou-se mais adequada ao modelo de Bem (1974). Dos vinte itens previstos para a escala feminina, 19 carregaram mais forte no fator 1 e o item 47 (fiel) carregou mais forte no fator 3. Dos vinte itens previstos para a escala masculina, 16 carregaram mais forte no fator 2 . Os itens 16 (liberal) e 28 (livre) não apresentaram carga em nenhum fator (considerando-se que foram excluídas as cargas fatoriais menores que 0,30). Os itens 19 (dominadora) e 31 (namoradora) apresentaram cargas maiores no fator 3. Dos vinte itens previstos para a escala neutra, 12 carregaram mais forte no fator 3 . Os itens 9 (sociável), 36 (espontânea), 39 (amigável) e 60 (esforçada) carregaram mais no fator 1 . Os itens 24 (otimista), 45 (coerente) e 51 (autodisciplinada) carregaram mais no fator 2. Por fim, o item 33 (organizada) não apresentou carga em qualquer fator (considerando que foram excluídas as cargas fatoriais menores que 0,30). Essas informações podem ser visualizadas na Tabela 1.

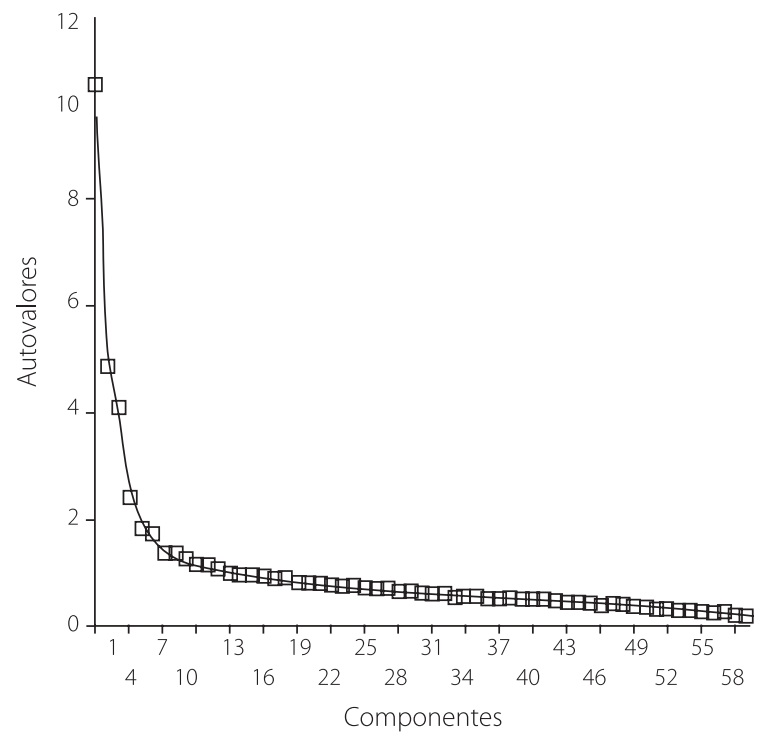

Figura 1. Scree plot dos fatores do BSRI extraídos pela Análise de Componentes Principais. 
Tabela 1. Cargas fatoriais e comunalidades dos itens do BSRI para uma solução de três fatores com rotação varimax. Porto Alegre (RS), 2005.

\begin{tabular}{|c|c|c|c|c|}
\hline \multirow{2}{*}{ Cargas fatoriais } & \multicolumn{3}{|c|}{ Escalas } & \multirow{2}{*}{ Comunalidades } \\
\hline & Feminina & Masculina & Neutra & \\
\hline 1 Valente & & 0,48 & & 0,24 \\
\hline 2 Romântica & 0,53 & & & 0,30 \\
\hline 3 Ponderada & & & $-0,41$ & 0,26 \\
\hline 4 Influente & & 0,54 & & 0,31 \\
\hline 5 Feminina & 0,56 & $-0,38$ & & 0,46 \\
\hline 6 Vulgar & & & 0,49 & 0,27 \\
\hline 7 Combativa & & 0,41 & & 0,20 \\
\hline 8 Prendada & 0,41 & & & 0,17 \\
\hline 9 Sociável & 0,41 & 0,30 & & 0,29 \\
\hline 10 Viril & & 0,38 & & 0,15 \\
\hline 11 Carinhosa & 0,58 & & & 0,35 \\
\hline 12 Invejosa & & & 0,50 & 0,26 \\
\hline 13 Arrojada & & 0,54 & & 0,30 \\
\hline 14 Vaidosa & 0,54 & & & 0,33 \\
\hline 15 Responsável & & & $-0,39$ & 0,23 \\
\hline 16 Liberal & & & & 0,10 \\
\hline 17 Emotiva & 0,58 & & & 0,34 \\
\hline 18 Leviana & & & 0,54 & 0,31 \\
\hline 19 Dominadora & & & 0,38 & 0,21 \\
\hline 20 Doce & 0,75 & & & 0,57 \\
\hline 21 Cínica & & & 0,52 & 0,27 \\
\hline 22 Atlética & & 0,39 & & 0,17 \\
\hline 23 Sonhadora & 0,35 & & & 0,16 \\
\hline 24 Otimista & 0,32 & 0,43 & & 0,31 \\
\hline 25 Líder & & 0,64 & & 0,41 \\
\hline 26 Dócil & 0,69 & & & 0,51 \\
\hline 27 Fofoqueira & & & 0,61 & 0,38 \\
\hline 28 Livre & & & & 0,14 \\
\hline 29 Delicada & 0,76 & & & 0,58 \\
\hline 30 Negligente & & & 0,53 & 0,28 \\
\hline 31 Namoradora & & & 0,48 & 0,29 \\
\hline 32 Sentimental & 0,62 & & & 0,40 \\
\hline 33 Organizada & & & & 0,12 \\
\hline 34 Competidora & & 0,56 & & 0,32 \\
\hline 35Terna & 0,60 & & & 0,38 \\
\hline 37 Masculina & 0,43 & 0,32 & & 0,29 \\
\hline 38 Charmosa & $-0,45$ & 0,51 & & 0,46 \\
\hline 39 Amigável & 0,50 & 0,33 & & 0,36 \\
\hline 40 Poderosa & 0,50 & 0,31 & & 0,36 \\
\hline 41 Caridosa & & 0,56 & & 0,39 \\
\hline 42 Queixosa & 0,43 & & & 0,23 \\
\hline 43 Galanteadora & & & 0,50 & 0,30 \\
\hline 44 Meiga & & 0,43 & 0,36 & 0,36 \\
\hline 45 Coerente & 0,77 & & & 0,59 \\
\hline 46 Autossuficiente & & 0,38 & & 0,28 \\
\hline $47 \mathrm{Fiel}$ & & 0,45 & & 0,22 \\
\hline 48 Mesquinha & & & $-0,32$ & 0,17 \\
\hline 49 Popular & & & 0,56 & 0,31 \\
\hline 50 Sensível & & 0,48 & & 0,25 \\
\hline 51 Disciplinada & 0,66 & & & 0,44 \\
\hline 52 Argumentadora & & 0,33 & & 0,27 \\
\hline 53 Suave & 0,75 & 0,45 & & 0,25 \\
\hline 54 Grosseira & & & 0,52 & 0,58 \\
\hline 55 Experiente & & 0,53 & & 0,33 \\
\hline 56 Graciosa & 0,71 & & & 0,30 \\
\hline
\end{tabular}


Tabela 1. Cargas fatoriais e comunalidades dos itens do BSRI para uma solução de três fatores com rotação varimax. Porto Alegre (RS), 2005.

\begin{tabular}{|c|c|c|c|c|}
\hline \multirow{2}{*}{ Cargas fatoriais } & \multicolumn{3}{|c|}{ Escalas } & \multirow{2}{*}{ Comunalidades } \\
\hline & Feminina & Masculina & Neutra & \\
\hline 57 Tagarela & & & 0,43 & 0,27 \\
\hline 58 Autoconfiante & & 0,54 & & 0,31 \\
\hline 59 Amável & 0,66 & & & 0,52 \\
\hline 60 Esforçada & 0,33 & 0,31 & $-0,30$ & 0,30 \\
\hline Autovalores & 10,1 & 4,9 & 4,1 & \\
\hline \% de Variância Explicada & 16,8 & 8,1 & 6,8 & \\
\hline Alfa de Cronbach & 0,90 & 0,81 & 0,61 & \\
\hline Média & 5,3 & 4,7 & & \\
\hline Desvio-padrão & 0,87 & 0,74 & & \\
\hline
\end{tabular}

Tabela 2. Comparativo entre estudos das médias (M), desvio-padrão (DP) e medianas (Mdn) dos escores de sexo dos sujeitos por escalas do BSRI. Porto Alegre (RS), 2005.

\begin{tabular}{|c|c|c|c|c|c|c|c|c|c|c|c|c|c|c|c|c|c|c|}
\hline \multirow{3}{*}{ Sexo } & \multicolumn{9}{|c|}{ Estudo de Hutz e Koller (1992) } & \multicolumn{9}{|c|}{ Estudo atual } \\
\hline & \multicolumn{3}{|c|}{$\begin{array}{c}\text { Escala } \\
\text { Feminina }\end{array}$} & \multicolumn{3}{|c|}{$\begin{array}{c}\text { Escala } \\
\text { Masculina }\end{array}$} & \multicolumn{3}{|c|}{$\begin{array}{l}\text { Escala } \\
\text { Neutra }\end{array}$} & \multicolumn{3}{|c|}{$\begin{array}{c}\text { Escala } \\
\text { Feminina }\end{array}$} & \multicolumn{3}{|c|}{$\begin{array}{c}\text { Escala } \\
\text { Masculina }\end{array}$} & \multicolumn{3}{|c|}{$\begin{array}{l}\text { Escala } \\
\text { Neutra }\end{array}$} \\
\hline & M & ${ }^{*} \mathrm{DP}$ & Mdn & M & ${ }^{*} \mathrm{DP}$ & $M d n$ & M & ${ }^{*} \mathrm{DP}$ & $M d n$ & M & ${ }^{*} \mathrm{DP}$ & $M d n$ & M & *DP & Mdn & M & *DP & Mdn \\
\hline Mulheres & 4,6 & & 4,6 & 4,2 & - & 4,2 & 4,2 & & 4,2 & 5,6 & 0,75 & 5,6 & 4,5 & 0,67 & 4,5 & 4,2 & 0,41 & 0,43 \\
\hline Homens & 4,0 & - & 4,0 & 4,7 & - & 4,7 & 4,1 & - & 4,1 & 4,9 & 0,86 & 5,0 & 5,0 & 0,70 & 5,1 & 4,1 & 0,45 & 0,42 \\
\hline Total & 4,3 & - & 4,3 & 4,4 & - & 4,4 & 4,1 & & 4,1 & 5,3 & 0,87 & 5,3 & 4,7 & 0,74 & 4,7 & 4,2 & 0,43 & 0,42 \\
\hline
\end{tabular}

* Dado não publicado pelos autores.

Tabela 3. Distribuição do sexo dos sujeitos por papéis sexuais classificados no estudo atual. Porto Alegre (RS), 2005.

\begin{tabular}{|c|c|c|c|c|c|c|c|c|}
\hline \multirow{2}{*}{ Sexo } & \multicolumn{2}{|c|}{ Feminino } & \multicolumn{2}{|c|}{ Masculino } & \multicolumn{2}{|c|}{ Andrógino } & \multicolumn{2}{|c|}{ Indiferenciado } \\
\hline & $n$ & $\%$ & $n$ & $\%$ & $n$ & $\%$ & $n$ & $\%$ \\
\hline Mulheres & 105 & 20,9 & 105 & 20,9 & 138 & 27,4 & 155 & 30,8 \\
\hline Homens & 74 & 17,7 & 72 & 17,2 & 126 & 30,1 & 147 & 35,1 \\
\hline Total & 179 & 19,4 & 177 & 19,2 & 264 & 28,6 & 302 & 32,8 \\
\hline
\end{tabular}

Da mesma forma que Hutz e Koller (1992), uma série de análises de variância Oneway foi executada para papéis sexuais e médias dos escores das escalas do BSRI. Foram encontradas diferenças estatísticas significativas nas escalas feminina, masculina e neutra: $F(3,918)=380$ $507, F(3,918)=343927$ e $F(3,918)=83890$, respectivamente. Todos os testes consideraram o nível de significância estatística de ( $p s<0,01)$ (Tabela 2).

O teste post hoc de Scheffé revelou que na escala feminina houve diferenças estatísticas significativas entre todos os tipos de papéis sexuais, exceto entre os típicos femininos e os andróginos; na escala masculina, houve diferenças estatísticas significativas entre todos os tipos de papéis sexuais, exceto entre os típicos femininos e os indiferenciados; na escala neutra, houve diferenças estatísticas significativas entre todos os tipos de papéis sexuais, sem exceção (Tabela 3).

Análises de variância para o fator papéis sexuais e itens do BSRI encontraram diferenças estatísticas significativas $(p<0,05)$ entre as médias de todos os itens, com exceção do 27 (fofoqueira) e do 42 (queixosa), ambos da escala neutra.

\section{Discussão}

Pode-se observar que, diferentemente dos resultados da pesquisa de readaptação (Hutz \& Koller, 1992), no estudo aqui relatado a escala feminina não 
apresentou grande dispersão de seus itens em outros fatores, já que 95\% tiveram cargas fatoriais mais elevadas no fator 1 (feminilidade). Por outro lado, os itens previstos para a escala masculina carregaram $80 \%$ no fator 2 (masculinidade) e 20\% no fator 3 (neutro), ou não apresentaram carga fatorial maior que 0,30.

Embora os itens da escala masculina tenham apresentado um desempenho fatorial razoável, é evidente que os da escala feminina foram muito melhores. Ao contrário do estudo de Hutz e Koller (1992, p.20), a escala feminina foi mais homogênea que a masculina. É possível que os resultados atuais possam ser compreendidos pelas mesmas explicações anteriores: "as mudanças culturais aceleradas não afetaram homens e mulheres da mesma forma".

Na mesma perspectiva, pode-se pensar que o item 47 (fiel), neste início do século XXI, não seja mais um atributo exclusivamente vinculado às mulheres. Talvez o crescimento da participação das pessoas do sexo feminino em diversos setores da sociedade leve-as a se igualarem aos homens no que diz respeito à característica fidelidade/infidelidade. Possivelmente, em alguns segmentos sociais já predomina a ideia de que a mulher é tão fiel/infiel quanto o homem. Pode-se inferir o mesmo em relação aos adjetivos livre, liberal, dominadora e namoradora, que não saturaram na escala masculina conforme o esperado. É importante registrar que dois dos quatro itens citados acima saturaram mais forte no fator 3 (neutro). Este fato acrescentaria mais evidências à tese do crescimento da igualdade dos sexos. Se isso for verdadeiro, deveria ser cogitada a possibilidade de substituição ou ajuste desses adjetivos nas escalas do BSRI.

Nenhum item previsto para a escala feminina apresentou carga maior na masculina e vice-versa, o que reforçou a ideia da independência e da estrutura fatorial bem definida do instrumento. Contudo, os itens da escala neutra não apresentaram o mesmo desempenho: 60\% carregaram mais forte no fator 3 (neutro) e 40\% dispersaram-se entre os fatores 1 (feminilidade) e 2 (masculinidade). Curioso que todos esses itens evadidos são de conteúdo positivo (socialmente desejáveis), e os itens de conteúdo negativo ficaram todos na escala neutra (fator 3 ).

Com relação à fidedignidade, os Alfas de 82 Cronbach calculados para a amostra superaram os coeficientes anteriores (Hutz \& Koller, 1992), principalmente para a escala feminina $(0,90)$. Para as escalas masculina e neutra, a diferença foi menor. Em suma, a avaliação atual constatou uma consistência maior do BSRI em comparação com a anterior.

\section{Considerações Finais}

$\mathrm{Na}$ consideração das diferenças encontradas entre os estudos comparados, deve-se considerar a diversidade entre a amostra usada no estudo de Hutze Koller (1992), exclusiva de estudantes universitários, e a atual; muito mais ampla e heterogênea em diversos aspectos (tais como escolaridade e idade). Além disso, decorreu aproximadamente uma década entre uma avaliação e outra. Essas duas condições diferenciadas talvez ajudem a explicar o melhor desempenho do BSRI no estudo atual.

Assim, considera-se que a adaptação brasileira do Bem Sex Role Inventory por Hutz e Koller (1992) mostrou-se válida e fidedigna nos primeiros anos do século XXI. Recomenda-se uma revisão meticulosa daqueles itens de desempenho fraco que sinalizam a necessidade de alguma atualização no instrumento.

\section{Referências}

Barberá, E. (1998). Psicología del género. Barcelona: Ariel.

Bem, S. L. (1974). The measurement of psychological androgyny. Journal of Consulting and Clinical Psychology, $42(2), 155-162$

Bem, S. L. (1977). On the utility of alternative procedures for assessing psychological androgyny. Journal of Consulting and Clinical Psychology, 45 (2), 196-205.

Bem, S. L. (1981). Gender schema theory: a cognitive account of sex typing. Psychological Review, 88 (4), 354-364.

Bussey, K., \&Bandura, A. (1984). Influence of gender constancy and social power on sex-linked modeling. Journal of Personality and Social Psychology, 47 (6), 1292-1302.

Cattell, R. B. (1966). The Scree Test for the number of factors. Multivariate Behavioral Research, 1 (2), 245-276.

Cheng, C. (2005). Processes underlying gender-role flexibility do androgynous individuals know more or know how to cope? Journal of Personality, 73 (3), 645-673.

Choi, N., \& Fugha, D. R. (2003). The structure of the Bem Sex Role Inventory: a summary report of 23 validation studies. Educational and Psychological Measurement, 63 (5), 872-887. 
Constantinople, A. (1973). Masculinity-femininity: an exception to a famous dictum? Psychological Bulletin, 80 (5), 389-407.

Fast, I. (1993). Aspects of early gender development: a psychodynamic perspective. In A. E. Beall \& R. J. Sternberg (Orgs.), The psychology of gender (pp.173-193). New York: Guilford Press.

Formiga, N. S., \& Camino, L. (2001). A dimensão do inventário de papéis sexuais (BSRI): a masculinidade e feminilidade em universitários. Estudos de Psicologia (Campinas), 18 (2), 41-49.

Gerrig, R. J., \& Zimbardo, P. G. (2005). A Psicologia e a vida. Porto Alegre: Artmed.

Gilbert, L. A. (1985). Measures of psychological masculinity and femininity: a Comment on Gaddy, Glass and Arnkoff. Journal of Couseling Psychology, 32 (1), 163-166.

Heerboth, J. R., \& Ramanaiah, N. V. (1985). Evaluation of the BSRI masculine and feminine items using desirability and stereotype ratings. Journal of Personality Assessment, 49 (3), 264-270.

Hiller, D. V., \& Philliber, W. W. (1985). Internal consistency and correlates of the Bem Sex Role Inventory. Social Psychology Quarterly, 48 (4), 373-380.

Hutz, C., \& Koller, S. (1992). A mensuração do gênero: uma readaptação do BSRI. Psicologia: Reflexão e Crítica, 5 (2), $15-21$

Jönsson, P., \& Carlsson, I. (2000). Androgyny and creativity: a study of relationship between a balanced sex-role and creative functioning. Scandinavian Journal of Psychology, 41 (4), 269-274.

Kaiser, H. F. (1960). The application of electronic computers to factor analysis. Educational and Psychological Measurement, 20, 141-151.

Kaiser, H. F., \& Rice, J. (1974). Little Jiffy, Mark IV. Educational and Psychological Measurement, 34 (1), 111-117.

Katsurada, E., \& Sugihara, Y. (1999). A preliminary validation of the Bem Sex Role Inventory in Japanese culture. Journal of Cross-Cultural Psychology, 30 (5), 641-645.

Kohlberg, L. (1972). Análisis de los conceptos y actitudes infantiles relativos al papel sexual desde el punto de vista del desarrollo cognitivo. In E. E. Maccoby (Org.), Desarrollo de las diferencias sexuales (pp.61-147). Madrid: Ediciones Marova.

Koller, S., Hutz, C. S., Vargas, S., \& Conti, L. (1990). Mensuração de gênero: reavaliação do BSRI. Ciência e Cultura, 42 (7), 517-518.

Konrad, A. M., \& Harris, C. (2002). Desirability of the Bem Sex-Role Inventory items for women and men: a comparison between African Americans and European Americans. Sex Roles, 47 (5-6), 259-271.

Lenney, E. (1991). Sex Roles: the measurement of masculinity, femininity, and androgyny. In J. P. Robinson, P. R. Shaver \& L. S. Wrightman (Orgs.), Measures of personality and social psychological attitudes (pp.573-660). San Diego: Academic Press.

Lott, B., \& Maluso, D. (1993). The social learning of gender. In A. E. Beall \& R. J. Sternberg (Orgs.), The psychology of gender (pp.99-122). New York: Guilford Press.

Maznah, I., \& Choo, P. F. (1986). The factor structure of the Bem Sex-Role Inventory (BSRI). International Journal of Psychology, 21, 31-41.

Oliveira, L. S. O. (1983). Masculinidadefeminilidade androginia. Rio de Janeiro: Achiamé.

Özkan, T., \& Lajunen, T. (2005). Masculinity, femininity, and the Bem Sex Role Inventory in Turkey. Sex Roles, 52 (1-2), 103-110.

Peng, T. K. (2006). Construct validation of the Bem Sex Role Inventory in Taiwan. Sex Roles: A Journal of Research, 55 (11-12), 843-851.

Reed-Sanders, D., Dodder, R. A., \& Webster, L. (2001). The Bem Sex Role Inventory across three cultures. The Journal of Social Psychology, 125 (4), 523-525.

Ross, L. D., Anderson, R., \& Wisocki, P.A. (1982). Television viewing and adult sex role attitudes. Sex Roles, 8 , 589-592.

Schmitt, B.H., \& Millard, R. T. (1988). Construct validity of the Bem Sex Role Inventory (BSRI): does the BSRI distinguish between gender-schematic and gender-aschematic individuals? Sex Roles, 19 (9-10), 581-588.

Spence, J. T., Helmreich, R., \& Stapp, J. (1975). Ratings of self and peers on sex role attributes and their relation to self-esteem and conceptions of masculinity and femininity. Journal of Personality and Social Psychology, 32 (1), 29-39.

Sternberg, R. J. (2000). Psicologia cognitiva. Porto Alegre: Artmed.

Twenge, J. M. (1997). Changes in masculine and feminine traits over time: a meta-analysis. Sex Roles: A Journal of Research,36 (5-6), 305- 326.

Ward, C., \& Sethi, R. R. (1986). Cross-Cultural validation of the Bem Sex Role Inventory. Journal of Cross-Cultural Psychology, 17 (3), 300-314.

Whetton, C., \& Swindells, T. (1977). A Factor analysis of the Bem Sex-Role Inventory. Journal of Clinical Psychology, 33 (1), 150-153.

Wilson, D., McMaster, J., Greenspan, R., Mboyi, L., Ncube, T., \& Sibanda, B. (1990). Cross-cultural validation of the Bem SexRole Inventory in Zimbabwe. Personality and Individual Differences, 11 (7), 651-656.

Zhang, J., Norvilitis, J. M., \& Jin, S. (2001). Measuring gender orientation with the Bem Sex Role Inventory in Chinese Culture. Sex Roles, 44 (3-4), 237-251.

Recebido em: 6/11/2006

Versão final reapresentada em: 9/5/2007

Aprovado em: 26/9/2007 